\title{
Los tecnodioses de la
}

\section{transparencia: el voyerismo del Adán digitalis en la cibersociedad pornóptica*}

\author{
[Artículos]
}

\author{
Edwin Hernando Alonso-Niño ** \\ Ciro Alejandro Ramírez-Cortés ***
}

Fecha de recepción: 4 de marzo de 2021

Fecha de aprobación: 18 de mayo de 2021

Citar como

Alonso-Niño, E. H. y Ramírez-Cortés, C. A. (2021). Los tecnodioses de la transparencia: el voyerismo del Adán digitalis en la cibersociedad pornóptica.

Via Inveniendi Et Iudicandi, 16(2). https://doi.org/10.15332/19090528.6786

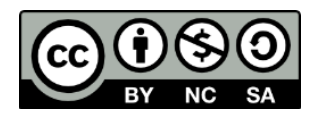

\section{Resumen}

La dialéctica ius-filosófico-teológica, que se pone de manifiesto en el presente manuscrito, es abordada desde cuatro dimensiones (quaestios): una dimensión antropológica, desde la cual, se presenta al hombre como

\footnotetext{
* Artículo producto del proyecto de investigación El Leviatán Inteligente: una Lectura Multidimensional del Derecho Administrativo frente a la Configuración de la Gobernanza Algorítmica en Colombia" de la Universidad Santo Tomas (Bogotá, Colombia).

** Doctor en Derecho con titulación honorífica cum laude por unanimidad de la Universidad Santo Tomás (Colombia). Magíster en Derecho Administrativo. Especialista en Derecho Administrativo. Abogado (cum laude). Docente investigador de la Universidad Santo Tomás (Bogotá, Colombia). Correo electrónico: hernandoalonso55@gmail.com, edwinalonso@usantotomas.edu.co. ORCID: https://orcid.org/0000-0001-5685-1718

*** Estudiante del Doctorado en Derecho, cohorte XI, de la Universidad Santo Tomás (Bogotá, Colombia). Abogado de la Pontificia Universidad Javeriana. Magíster en Gerencia Pública de la Universidad de los Andes. Senador de la República de Colombia. ORCID: https://orcid.org/0000$\underline{0002-7438-1200}$
}

Via Inveniendi Et Iudicandi

e-ISSN: 1909-0528 | DOI: https://doi.org/10.15332/19090528

Vol. 16 N.0 2 | julio-diciembre del 2021 
un ser humano que se gestó y desarrolló en los cauces de la racionalidad del Homo sapiens, pero, en la actualidad, pretende evolucionar y dar lugar al Homo digitalis. Dicha idea permite el desarrollo de una dimensión ética, desde la cual se justifica la pregunta central que atraviesa la investigación y que, de manera específica, pretende indagar por cuál es la idea de hombre que subyace y se construye a partir de la configuración de un Homo digitalis influenciado por la inteligencia artificial (IA).

Con el fin de sustentar la disputatio, se aborda una dimensión de sentido, cuestión desde la que se presenta al hombre como un constructo que se encuentra expuesto (desde la dialéctica del pornóptico digital), vigilado de manera constante e imbuido en las redes de la IA, pero, su comportamiento "virtual" le lleva a la renuncia, casi involuntaria, de ciertas libertades y derechos que presume dominar (y que rompe el esquema de un constitucionalismo clásico). Finalmente, como última dimensión, que resultaría siendo holística al desarrollo que antecede, queda expuesta una síntesis de saberes como razón ampliada, en virtud de la que se reconoce a un hombre con pretensión de tecnificación, que a la vez es cosificado (voyerista) por la misma IA y seducido por la manzana de la virtualidad que, de manera vertiginosa, digiere su racionalidad humana.

Palabras clave: Homo sapiens, Homo digitalis, inteligencia artificial, voyerismo, pornóptico.

\section{The techno-gods of transparency: the voyeurism of Adam digitalis in the pornoptic cybersociety}

\section{Abstract}

The ius-philosophical-theological dialectic, which is highlighted in this manuscript, is approached from four dimensions (quaestios): an anthropological dimension, from which man is presented as a human being that was gestated and developed in the channels of the rationality 
of Homo sapiens, but at present, aims to evolve and give rise to Homo digitalis. This idea allows the development of an ethical dimension, from which the main question that runs through the research is justified and that, specifically, seeks to investigate what is the idea of man that underlies and is built from the configuration of a Homo digitalis influenced by artificial intelligence (AI).

In order to support the disputatio, a dimension of meaning is addressed, an issue from which man is presented as a construct that is exposed (from the dialectic of digital pornoptic), constantly supervised and imbued in the networks of AI, but his 'virtual' behavior leads him to the renunciation, almost involuntary, of certain freedoms and rights he presumes to dominate (and that breaks the scheme of a classic constitutionalism). Finally, as the last dimension, which would turn out to be holistic to the previous development, a synthesis of knowledge is exposed as an extended reason, by virtue of which a man with claims of technification is recognized who, at the same time, is objectified (voyeuristic) by the AI itself and seduced by the apple of virtuality which, in a vertiginous way, digests its human rationality.

Keywords: Homo sapiens, Homo digitalis, artificial intelligence, voyeurism, pornoptic.

Sabremos cada vez menos qué es un ser

humano...

Saramago (2006)

\section{Introducción}

La extinción del Homo sapiens para dar nacimiento al Homo digitalis es una realidad. La tentación de amplificar la inteligencia y los deseos en un proceso de hibridación con las máquinas está a la orden del día, y es, en este contexto, en el que se inscribe al siglo XXI. Así las cosas, al Adán digitalis se le ha presentado una "serpiente tecnológica" que le ha 
ofrecido una manzana que tiene forma de virtualidad y de inteligencia artificial (IA).

$\mathrm{Al}$ comer insaciablemente de la manzana de la virtualidad, el Adán digitalis debe pagar un precio: perder su alma, tecnificar sus sentimientos, esconderse de sus propios errores. En este contexto, el paraíso de la virtualidad desdibuja la esencia misma del ser humano y les ofrece a los dioses tecnológicos (tecnodioses) un sacrificio: el libre albedrío. Pero ¿qué empuja al extinto Homo sapiens a esta tragedia?: sencillamente la soberbia de querer o pretender ser más que Dios (Harari, 2014). El ánimo de querer tener siempre más no implica necesariamente un proceso evolutivo que pudiera siquiera ser equiparable al clásico modelo darwinista. Así las cosas, lo que se presenta y viene es la absorción de la racionalidad humana por mecanismos de inteligencia artificial (IA), en los que dicha racionalidad es digerida. Bajo este panorama, se cuestiona, en el contexto de globalización, ¿qué idea de hombre subyace y se construye bajo patrones de vigilancia tecnológica y adoctrinamiento social que impone la IA?

La tesis central que permite responder anticipadamente a la pregunta precedente, y que se erige además como eje de navegación en este texto, permite afirmar que desde una sociedad de vigilancia tecnológica (Bauman y Lyons, 2013) que imprime patrones de adoctrinamiento social, se configura un prototipo de hombre individualista y limitado en su libertad. En efecto, este tipo de Homo digitalis crea vulnerabilidades a nivel social, de hecho, permeando espacios que el mismo Estado no logra proteger (Bobbio, 1989), configurándose una nueva manera de dominación (Alonso, 2002) que termina por reproducir un sistema que es dominante a través de un control y adoctrinamiento psicológico en el que se involucran 
varios actores que fijan y modelan su conducta, sin ser muchas veces conscientes de ello en su totalidad (Bourdieu, 2011).

El mundo debe ser consciente de que "una mutación sin precedentes está ocurriendo en la vida de los hombres" (Wajcman, 2011, p. 13). Así, los fantasmas kafkianos se han transformado (Kafka, 1998) y con ello las formas de interacción social que son impactadas por la tecnología cuestionan, hoy en día, la esencia misma del ser humano, que, desde una inconsciencia voluntaria, se exhibe y expone ante una sociedad de la vigilancia y el control. Los nuevos fenómenos tecnológicos han dado lugar a que desaparezcan esferas privadas de la vida humana para dar paso a una vertiginosa comunicación digital que se torna casi incontrolable, donde la inestabilidad y las amorfas relaciones sociales resultan siendo impersonales (Arendt, 2002).

La manzana de la virtualidad implica una convivencia en una sociedad del irrespeto. Si bien, el respeto (respectare) implica una visión distanciada (pathos de la distancia), en una realidad como la presente, dicha situación configura un típico especatáculo (spectare). Así, la delgada línea diferencial entre el respectare y el spectare que imprime la interacción social desde las dinámicas de las tecnologías de la información y la comunicación (Moreno y Velásquez, 2015) permitirían presentar a una sociedad que carece de pathos de la distancia; que conduce necesariamente a una sociedad del escándalo, a una sociedad de la indignación (Han, 2014a).

Si bien el respeto se erige como elemento clave de lo público, se debe reconocer que el desaparecimiento de aquel implica la decadencia de este último. Así, la decandencia y falta de respeto son variables relacionadas de manera interdependiente, de manera que en la sociedad actual impera una completa falta de distancia en la que la intimidad se expone de forma 
deliberada y sin mayor decoro. Así, la comunicación permeada por lo digital, pese a permitir desdibujar las distancias físicas, se conjuga con una completa erosión mental que ubica a la población en un contexto de riesgo.

En la sociedad del siglo XXI, el Homo digitalis reconoce en la tecnología una especie de dependencia farmacológica, en la que el uso de las tecnologías y la manera como estas actúan en el ser humano crean nuevos dominios de saber que moldean al invididuo de formas no conocidas con antelación. Entonces, debe reconocerse una estructura social hiperconectada, aparentemente libre, que transforma modos de generación de riqueza, "autoregulándose" de acuerdo con ciertas necesidades del mercado.

Dicha hiperconectividad causa cambios sustanciales en las formas de interacción de los sujetos involucrados en la dialéctica denominada sociedad. Los nuevos mecanimos de comunicación, que estructuran al homo-digitalis, le transforman y le someten a formas de poder "invisibles" que le disciplinan, pero que, a su vez, le vigilan constantemente. Un ejemplo claro de lo expuesto, entre otras cosas, se ubica en las redes sociales $^{1}$, de manera que, a través de estas, se crean bases de datos con detallada información de los individuos que allí interactúan, formando, desde dichas bases, patrones de comportamiento (Galvis y Pesca, 2020). Así las cosas, fijando un contexto de actualización a los clásicos planteamientos de Foucault (2002), bien podría presentarse la configuración y existencia de un panóptico que ha hecho tránsito hacia lo digital (panoptismo digital), que guarda información de todos los sujetos a

\footnotetext{
${ }^{1}$ Las redes sociales son un ejemplo incuestionable en lo que refiere a la transparencia personal. En las redes sociales se torna, de alguna manera, difícil mentir. Verbigracia, en una red como Linkedin no es posible mentir, ya que depende de la información verídica que el resto de Homo digitalis puedan valorar el currículum y, de allí, obtener alguna vinculación laboral. Por esta razón, se crea una sociedad de la transparencia, en la que todo lo que se comparte "voluntariamente" en internet es susceptible de la mirada de todo el mundo.
} 
nivel global (Baker, 2009) y que, a su vez, configura a una sociedad "psicopolítica" de la transparencia, en la que se introduce un claro psicopoder por medio de la vigilancia digital, fenómeno este que adquiere la capacidad, no solo de vigilar, sino de controlar y mover a las personas desde su mismo componente intrínseco, llegando a dominar, en términos de masa social, su misma lógica de interacción (Parsons, 1952).

El contexto social, donde se desenuelve el Adán digital, implica concebirlo en la dinámica de la evolución panoptista que se ha venido fraguando. Un campo donde la información se utiliza de manera indiscriminada y en el que la consecuente sociedad de la transparencia que toma forma convive bajo patrones de supuesta libertad y control que resultan siendo ejercidos de manera invisble (Koerner y Perafán del Campo, 2020).

En la vigilancia digital, los vigilantes somos todos, es decir, coexiste reciprocidad en la vigilancia de cada uno de los individuos y estos, a diferencia del individuo disciplinado que ejercía resistencia ante la coacción del modelo de vigilancia orwelliano (con su telepantalla y cámaras de tortura), en el modelo del panóptico digital encuentra fascinación por la intimidad del otro y por mostrar lo más profundo de su ser, gracias a su hipercomunicación y consumo (Vitalis, 2015).

Entonces, el voyerismo se erige como parte fundamental de las modernas relaciones sociodigitales en las que las redes sociales, los smartphones, las Google Glass, entre otros tecnodioses, terminan dominando la apariencia de libertad y la comunicación sin fronteras. En esta sociedad transparente no existe una tortura per se, pero sí se postea, tuitea o se (des)aprueba a través de un like; elementos que cambian la forma de "confesarse" ante el mundo, ya que, en lugar de suplicios y vejámenes, lo que se pone a la 
orden del día es un completo desnudamiento voluntario, de suerte que los aparatos digitales ${ }^{2}$ sustituyen a la cámara de tortura.

De acuerdo con lo expuesto, se encuentra, por un lado, que la exhibición pornográfica de datos e información privada y el control panóptico, por otro lado, se compenetran (Barba y Montes, 2007). El voyerismo digital exhibicionista alimenta a las redes que adquieren forma de panóptico moderno, donde la sociedad objeto de control no se desnuda en virtud de algún tipo de coacción, sino todo lo contrario, es decir, se hace bajo la premisa de una aparente libertad en la que la necesidad de "mostrarse" a los otros cede sin problemas en el vertiginoso mundo tecnológico. Al respecto, McLuhan (1996), teórico de los medios, sostenía, a manera de advertencia, en 1964, que "la tecnología eléctrica ya está dentro de nuestros muros y estamos embotados, sordos, y mudos ante su encuentro con la tecnología de Gutenberg” (p. 87).

Queda en evidencia el declive de múltiples valores ético-sociales y humanos entre ellos, la misma intimidad del individuo. El ejercicio indiscriminado de aquella está llevando paradójicamente a la vorágine de la racionalidad y pareciera ser que lo que en principio parecía ser prometedor desde la perspectiva de lo virtual, con el pasar del tiempo adquiere más las características de un leviatán.

\section{La manzana de la virtualidad: episteme de la filosofía tecnológica frente al Adán digitalis}

La serpiente tecnológica que se mueve vertiginosamente en el mundo y ofrece múltiples bondades al Adán-digitalis, le hibrida con la virtualidad, pero le aleja de elementos fundantes de la existencia humana, encontrando

\footnotetext{
${ }^{2}$ Según Han (2014a) son considerados como gadgets de silicio entendiendo que "los aparatos digitales traen una nueva coacción, una nueva esclavitud (voluntaria)".
} 
nuevos dioses en los aparatos electrónicos y en las redes que se tejen en lo digital.

El entendimiento de la tecnología ha sido dinámico. Por un lado, suele concebirse como un conjunto de saberes que permiten la conquista de un fin determinado; pero, por otro, aristotélicamente, puede responder a un conjunto de conocimientos científicos que se ordenan de manera técnica (téchne) para la satisfacción de necesidades y deseos humanos. Así las cosas, el uso de la téchne digital trae consigo ventajas, pero también puede traer desventura, ya que su uso es lo que provoca la destrucción del Homo sapiens, sin desconocer que su uso racional puede contribuir al desarrollo social e individual. Entonces, el uso de lo digital puede ser positivo o negativo, puede cosificar, alienar, marginar o, en contraposición, puede potenciar al progreso, al bienestar y al equilibrio.

Lo anterior permite inferir que a los Homo digitalis no les convoca mayor interés por la reflexión sobre la esencia misma del acto tecnológico, ya que las preocupaciones inmediatas están centradas en la toma de decisiones capitalistas que redundan en el cómo o cuándo se puede adquirir el aparato electrónico deseado, pretendiendo soslayar la marginación social que ha impuesto la revolución tecnológica.

Entonces, la realidad de lo digital se presenta como reduccionista, sobre todo cuando en su esencia no se encuentran insertas nociones más amplias como la misma naturaleza humana, concepto a su vez inseparable de la idea de Dios. Por ello, uno de los cometidos del filósofo tecnológico es la aprehensión del ser, de su sentido y de su significado fenomenológico que le articula con la misma teología existencialista, resultando insoslayable separar la tríada ántrophos, paideia, theos; pues las tres categorías son parte de una misma realidad histórica y sociocultural. 
Abordar a la tecnología como eje estructurante de esta reflexión implica entender que esta, filosóficamente hablando, involucra la formulación de problemas y cuestiones fundamentales a saber: en primer lugar, se ubica un problema ontológico desde el que se pretende responder al ser, su esencia y a la existencia de la tecnología propiamente dicha.

Por otro lado, el problema gnoseológico buscaría resolver asuntos como el origen, la esencia, verdad y límites del campo tecnológico, puesto al servicio del Homo-digitalis; presentando a este como sujeto cognoscente frente a un objeto cognoscible. De manera seguida, el problema epistemológico se preocuparía por indagar sobre los paradigmas tecnológicos y su estructuración interna, analizando su articulación con la ciencia. Por parte del problema axiológico existiría el cuestionamiento sobre la operativización de los valores al servicio de la tecnología, relacionando lo anterior con el problema teleológico que se cuestiona sobre los fines de esta.

Así las cosas, los retos digitales que se le imponen al Adán digitalis le ubican en una serie de campos problémicos donde la tecnología, si bien se presenta como novedosa, en la época en que surge es vista como una forma de superar lo clásico, está limitada temporalmente, pues cada que se avance históricamente, se presentará como insuficiente de acuerdo con las exigencias de contexto.

De lo anterior se logra comprender la génesis del campo de la tecnología, entendiendo que no se trata de un mero factum de inicio, sino que se trata de una téchne que transita por un camino que en principio no tiene fin. Ello, justamente, da como resultado una nueva manera de concebir y transformar al mundo, presentando nuevas maneras de entendimiento del entorno, creando mecanismos diversos para instalarse o ubicarse en dicho 
mundo, de manera que el Homo digitalis se erige como el principal agente de mediación histórica entre la tríada: pasado, presente y futuro.

Dicha mediación implica un dialecto entre lo subjetivo y lo objetivo, entre lo conocido y lo cognoscible, entre la naturaleza y el ser humano. Es por esto que la tecnología y su filosofía han de combinar, de manera simbiótica, teoría y praxis; siendo esta una realidad dinámica y a la vez compleja, en la cual, sin importar el momento en que se presente el interés cognoscitivo del filósofo tecnológico, este siempre deberá optar por proporcionar una visión del presente.

En aras de fijar un acercamiento a la realidad del conocimiento y praxis tecnológica, es valioso, desde una perspectiva fenomenológica, comprender la manera como el Adán digitalis se enfrenta y relaciona con la realidad virtual; situación que permite comprender que la serpiente de la tecnología, en sí misma, se yuxtapone como una realidad temporal que va sustituyendo, entre múltiples factores: formas de ser, pensar, sentir; incluso, el mismo desarrollo de personalidades mucho más autónomas, pero, a la vez, autómatas.

Por ende, el binomio Homo digitalis y tecnología constituyen en sí mismo un problema, como quiera que el ser humano es inacabado, imperfectum, siendo un proyecto en constante construcción, sucediendo lo pertinente con lo digital que, visto desde una globalidad histórica, se presenta como un constructo interminable natural a la especie humana. De allí que tanto el ser humano, visto desde lo digital, así como la tecnología, dan cuenta de problemas constantes y perpetuos, donde se recorren sendas análogas y una infinidad de caminos con variados obstáculos que pretende superar. A manera de síntesis, bien podría sostenerse por el momento la importancia para que el ser humano ejecute un uso racional del campo tecnológico que no le limite, le cosifique o, peor aún, le instrumentalice

Via Inveniendi Et Iudicandi

e-ISSN: 1909-0528 | DOI: https://doi.org/10.15332/19090528

Vol. 16 N. 02 | julio-diciembre del 2021 
siendo el artífice de su propia destrucción. Bajo esta idea, resulta valioso comprender la realidad fenomenológica en la que se desenvuelve nuestro Adán digitalis, que, de manera voluntaria, pero inconsciente, se expone, exhibe y muestra ante una sociedad permeada por la tecnología, haciendo del voyerismo digital su estilo de vida.

\section{El voyerismo y los hikikomoris tecnológicos: un estilo de vida propio del Adán digitalis ante el panóptico moderno}

Es valioso comprender la fenomenología relacional del Adán digitalis con la realidad tecnológica a la luz de las prácticas e implicaciones que demarca su actuar que trasciende a lo público. Hoy, el Adán digitalis vive en un "mundo de lo no muerto, en donde no solo se ha hecho imposible nacer, sino también morir" (Saramago, 2006). La tecnología, lo digital y virtual le exhiben exponencialmente ante el mundo, de manera tal que parece casi imposible existir o dejar de hacerlo sin dejar rastro alguno. Esa, sencillamente, es la consecuencia de la datificación y la acción del Homo digitalis, quien, desde su premisa de libertad en el mundo de la web, queda expuesto ante una sociedad de la transparencia.

Contrario a lo que sucede con un espectador en el estadio deportivo, el Homo digitalis es cualquier cosa, excepto nadie. Pretende manifestarse de forma incógnita, pero es frecuente verle con un perfil en el que trabaja de manera insaciable para "actualizarlo" y, en vez de ser un ser anónimo, se presenta como un vector de penetración y exposición social que reclama atención.

La identidad del Homo digitalis se diluye en la red, desvaneciendo la órbita de lo privado para dar paso a un campo predominantemente colectivo. En este sentido, McLuhan (1978) comprende que el hombre de 
masas $^{3}$ habita como una especie de morador electrónico que puede estar unido con más habitantes, pero donde su identidad privada y personal se ve extinguida por una excesiva carga de vida digital.

Entonces, en el contexto expuesto, el Adán digitalis, a pesar de estar en "redes", que en apariencia conectan, en verdad no se congregan, es decir, existe una colectividad de usuarios sin identidad común, sin congregación, vale decir, un conjunto sin alma o espíritu, convirtiéndose en hikikomoris 4 aislados, individualistas, con sentimientos de soledad ante el display, de manera que los modelos de movimiento social se tornan fugaces e inestables (inter)actuando de manera carnavalesca, lúdica y no vinculante, donde la (des)aprobación de muchas facetas de la vida, que se pensaría íntimas, se reducen a una "feria de likes" que pareciera legitiman la toma de muchas decisiones al interior de las multitudes inteligentes -smart mobs-.

El Homo digitalis, como hikikomoris, habita en una sociedad que se encuentra expuesta a un gran volumen de información, que aumenta de manera veloz y se multiplica sin control en la web. En este aspecto, lo que cada persona sube a la red bajo la presentación de un "perfil privado", termina siendo público y de conocimiento colectivo a pesar de encontrarse "agregados", la realidad es que hay algún tipo de existencia en la red (Han, 2014b).

Dicho aumento exponencial de la información ha sido objeto de estudio por parte del norteamericano Lewis (1996), para quien, desde el terreno de

\footnotetext{
${ }^{3}$ Gustave Le Bon (1895) define a la modernidad como la "época de las masas", expresando que "el presente, dice, es un periodo de transición y de anarquía" (p. 45). Y constata, de forma lacónica: "la era en la que entramos será, verdaderamente, la era de las masas" (p. 46).

4 "Personas que viven al margen de la sociedad. Por ejemplo, alguien que se pasa el día entero ante los medios audiovisuales, apenas sin salir de casa" (Han, 2014b).
} 
la psicología, aquel fenómeno se enmarca en lo que se conoce como la Information Fatigue Syndrom (IFS).

Desde sus planteamientos, dicho trastorno es generado por una sobrecarga en la información y los afectados por dicho síndrome reportan, de manera creciente, parálisis en su capacidad analítica, lo cual trae aparejada una evidente afección en la capacidad de atención y en el sentido de asumir responsabilidades. Hoy, debido a la oleada digital, la población está afectada, de alguna forma, por el IFS, lo cual está causado por la vertiginosa cantidad de información que transita en el mundo de lo digital. El exceso de información hace que se atrofie la capacidad racionalanalítica que es la esencia constructiva del pensamiento, sobre todo, porque la capacidad analítica consiste en prescindir de todo aquello lo que no pertenece, en esencia, a alguna cosa. El diluvio de exposición a la información con la que se cuenta en la actualidad, sin duda, afecta y disminuye la capacidad de reducción a lo esencial.

El IFS incluye también síntomas connaturales a esta patología, como la depresión, por más narcisista que se le califique. En todo caso, la modulación que del mundo hace, el Adán digitalis le lleva a un mar de depresión cuando no se reconoce a sí mismo de alguna forma y donde depende de que el mundo exterior le (des) "apruebe" sus conductas existencialistas en el "muro de los lamentos" digitales (Facebook, Instagram, Twitter, entre otros).

Un like, amén digital en la sociedad moderna, se presenta como un insumo de (des)aprobación existencialista que se relaciona e involucra directamente con la misma exposición social que el mismo Adán digitalis ha generado. Así, la vigilancia del "otro" se configura como una práctica habitual que termina por justificar un voyerismo que supera al modelo de control benthamiano y owerlliano de coacción suplicio físico.

Via Inveniendi Et Iudicandi

e-ISSN: 1909-0528 | DOI: https://doi.org/10.15332/19090528

Vol. 16 N.0 2 | julio-diciembre del 2021 
Recuérdese que el panóptico de Bentham (2011) era presentado como un modelo de sociedad disciplinaria y correccional, un control que, en su forma tradicional de concepción, se aplica y somete a instituciones como la familia, los manicomios, las cárceles, los cuarteles militares, las empresas, los hospitales, las escuelas (sociedades todas netamente disciplinarias). La "torre de control” que es característica del modelo panoptista clásico conlleva, además de la vigilancia constante, la imposibilidad de un libre desarrollo de la personalidad, so pena de ciertos castigos y correcciones propias del modelo disciplinario al que se hace alusión. Lo particular es que el vigilante, en este modelo, tiende a permanecer oculto o invisible a los vigilados, ya que: "la esencia de esto consiste en la centralidad de la situación del inspector, combinada con las bien conocidas y más efectivas tretas de ver sin ser visto" (Bentham, 2011, p. 54).

A diferencia del modelo panoptista clásico, los moradores del actual panóptico digital, mantienen una comunicación constante y un exhibicionismo permanente, en el que se hace uso intensivo de la libertad, que resulta posible, debido a que, de forma voluntaria, pero también sin plena consciencia, tiene cabida un desnudamiento propio.

Entonces, el big brother digital sobrepasa la coacción a la que están sometidos bajo el modelo clásico los reclusos, tornándose más eficiente su nueva cara y de ahí la gran diferencia, es decir, mientras los moradores del panóptico de Bentham saben de manera racional de la presencia del vigilante, la cual es constante; por su parte, quienes conviven en el panóptico digital creen estar en total libertad.

El panoptismo ha alcanzado un nivel de expansión masivo hasta el punto de referirse al panoptismo digital como un nuevo concepto que involucra las redes sociales y el mundo cibernético. El internet se ha adentrado como un gusano en la manzana, en las dinámicas sociales, económicas y

Via Inveniendi Et Iudicandi

e-ISSN: 1909-0528 | DOI: https://doi.org/10.15332/19090528

Vol. 16 N.0 2 | julio-diciembre del 2021 
políticas, hasta convertirlo en una constante a la cual se recurre para explicar los comportamientos de los nodos de comunicación virtual. El actual sistema está planeado para que la información llegue a todos los rincones del mundo, para que conozcamos más de los demás e incluso como recurso del sistema político, el cual participa como un "gran hermano" virtual.

La particularidad del panóptico digital está especialmente en que sus habitantes son muy colaboradores y activos en su construcción y mantenimiento, exhibiéndose de manera voluntaria y voyerista. En este mercado, la exhibición pornográfica datificada y el consecuente control panoptista se compenetran (Bukowski, 1978). En este contexto, no se puede desconocer la presencia de serpientes voraces que alimentan las redes como panóptico.

El proyecto del panóptico de Bentham tiene una motivación sobre todo biopolítica. Uno de los primeros efectos que se deberían esperar del panóptico y su control es la "reforma" o modulación de las costumbres. Hoy el mundo se desarrolla en el marco de un panóptico de grandes magnitudes en el que, las aparentes formas de libertad adoptan formas panópticas (Assange, 2014).

En la actualidad, la vigilancia y el control no se presentan como ataques frontales a la libertad, sino que, de hecho, esta se cede o entrega de manera voluntaria a la mirada de los espectadores. Entonces, a sabiendas, el Adán digitalis contribuye al panóptico digital, en la medida en que se desnuda y se expone, siendo víctima y actor a la vez; programándose de forma autómata sin captar el cambio radical de paradigma, que cambia, de manera decisiva, la conducta, las percepciones, las sensaciones y sentimientos, los pensamientos, la convivencia misma. 
Bien podría afirmarse, a la luz de lo expuesto, que el Adán digitalis se embriaga hoy con el medio digital, sin poder valorar por completo las consecuencias de su ebriedad, de suerte que la ceguera ante el paradigma actual y la simultánea obnubilación 5 constituyen la crisis actual. Allí radica la dialéctica libertaria que se patentiza como forma de control.

Dicha crisis viene dada por la mediación de lo digital que, como bien se expuso antes, es perjudicial para el respeto que deontológicamente se pretende en sociedad, generando seres individualistas a manera de Ádyton ${ }^{6}$. En este contexto, lo digital implica que se fomente la propagación pornográfica de lo privado, sometiendo y moldeando realidades que ahora transitan entre lo imaginario y simbólico7.

Hoy, los dispositivos tecnológicos (como los smartphones) hacen las veces de espejos digitales que abren estadios narcisistas y, como se acaba de exponer, propician lo imaginario (por ejemplo, cuando se generan limitaciones frente a las suposiciones causadas como consecuencia de un chat).

El Adán digitalis se mantiene desnudo y sin conciencia de ello, pese a la constante tentación de la serpiente tecnológica. Así, sube al mundo de las redes todo en cuanto le identifique, desconociendo en qué latitudes se puede llegar a saber de él. Su paraíso parece ser de otro mundo, el digital

\footnotetext{
${ }^{5}$ Este fenómeno obedece a un trastorno de la conciencia en el que la capacidad de estar alerta y vigilante se disminuye. Es una especie de estado letárgico con baja capacidad de reacción a estimulaciones externas.

${ }^{6}$ Ádyton: corresponde al espacio completamente cerrado (hacia fuera) en el templo griego.

${ }^{7}$ En un ensayo sobre el décimo aniversario de Skype puede colegirse que el medio digital nos aleja cada vez más del otro, resultando acertado referenciar: "El videoteléfono produce la ilusión de una presencia y sin duda ha hecho más soportable la separación espacial entre amantes. Pero se nota siempre la distancia, que permanece, quizá con la mayor claridad en una pequeña descentración. Efectivamente, en Skype no es posible mirarse el uno al otro. Cuando en la pantalla se mira a los ojos del otro, este cree que su interlocutor mira ligeramente hacia abajo, pues la cámara está instalada en el marco superior del ordenador. La bella peculiaridad del encuentro inmediato, la de que ver a alguien es siempre equivalente a ser visto, ha dejado paso a la asimetría de la mirada. Gracias a Skype, podemos estar cerca los unos de los otros las veinticuatro horas del día, pero dejamos constantemente de mirarnos" (Skype, 2013).
}

Via Inveniendi Et Iudicandi

e-ISSN: 1909-0528 | DOI: https://doi.org/10.15332/19090528

Vol. 16 N.0 2 | julio-diciembre del 2021 
justamente, donde la seducción de la serpiente ya no le representa una expulsión, sino, todo lo contrario, una especie de inclusión que se genera a propósito del resto de moradores tecnológicos. Esta exposición del Adán digitalis deja como obsoleta la noción misma de protección de datos, transitando al campo de la psicopolítica digital, que implica un tránsito de una vigilancia pasiva a un control activo.

Entonces, el campo digital del Adán moderno, que se desarrolla en el contexto del big data, se presenta como herramienta psicopolítica y de gran eficiencia dominadora que, incluso, permea la psique y le condiciona a niveles prerreflexivos y autómatas. De alguna manera, el futuro de los moradores digitales es controlable y predecible, generando proyecciones de ansiedad, entre tanto se espere el cumplimiento de lo que la tecnología ofrece, de suerte que el big data es una especie de sentencia que anuncia la extinción de la persona humana y la libertad (Greenfield, 2015).

En este contexto de dominación se generan objetos de devoción que tienen la capacidad de someter. Por ejemplo, el smartphone, al que se ha hecho referencia, se configura como un dispositivo de completa devoción y subjetivación que hace las veces, para creyentes y no, de una clase de "rosario" que se presenta a la vez como una especie de móvil. Este tiene la capacidad de atribuirles a ambos la posibilidad de examinarse y controlarse a sí mismo. En todo caso, la dominación aumenta su eficacia cuando delega a cada uno la vigilancia. El smartphone, entre otros dispositivos tecnológicos, no solo actúa como eficiente aparato de vigilancia, sino también como práctico confesionario móvil, pues, por ejemplo, las redes sociales se erigen como iglesias o una especie de sinagoga mundial.

En suma, en el mundo del Adán digitalis lo que se configura es una congregación de lo digital, que posee registro de cada clic que se hace, 
de cada palabra que se introduce. En general, todo paso en la red es vigilado y registrado ${ }^{8}$, reproduciéndose, totalmente, la vida (y su estilo) en la red. Los hábitos digitales proporcionan representaciones exactas de la personalidad, de hecho, puede que más acertadas que la misma imagen que cada uno tiene de sí mismo (basta con consultar la perfilación de cada uno en buscadores como Google para encontrar una detallada descripción de gustos, intereses, hobbies y, en general, el estilo de vida y clase de persona).

\section{El confesionario digital: las falsas emociones en el pornóptico del Adán digitalis}

Como ha quedado expuesto, los dispositivos electrónicos en el mundo moderno se presentan como prácticos confesionarios móviles que permiten, en el muro de los lamentos de la red social que corresponda, volcarse en confesión, justificando sus emociones. En consecuencia, obteniendo la (des)aprobación de la comunidad internacional (Huxley, 2013).

Hoy se habla de sentimiento o de emoción de una manera reciente y, de hecho, en múltiples campos las emociones son objeto de investigación. De repente, el ser humano no es solo un animal rationale, sino que también es un ser de sentimientos. Bajo este entendido, el marketing y el capitalismo de consumo (emotional design) introducen emociones tendientes a estimular la compra y generación de "necesidades" (muchas veces innecesarias).

\footnotetext{
8 "El número de direcciones web es prácticamente ilimitado. De este modo es posible dotar a cada objeto de uso de una dirección en internet. Las cosas se convierten por sí mismas en proveedores activos de información. Informan sobre nuestra vida, sobre nuestro hacer, sobre nuestras costumbres. La extensión del internet de las personas, web 2.0, al internet de las cosas, web 3.0, es la culminación de la sociedad de control digital. La web 3.0 hace posible un registro total de la vida. Ahora también nos vigilan las cosas que usamos diariamente" (Han, 2014a).
}

Via Inveniendi Et Iudicandi

e-ISSN: 1909-0528 | DOI: https://doi.org/10.15332/19090528

Vol. 16 N.0 2 | julio-diciembre del 2021 
Entonces, el Adán digitalis está atrapado en una memoria total de tipo digital, en la que nada es objeto de olvido y justamente esta es la practicidad del big data que, desde la lógica de un big brother orwelliano, el digital es mucho más eficiente (Han, 2014a).

De acuerdo con lo expuesto, el Adán digitalis, que está expuesto, no solo al big data, sino también al big deal (mercado), se somete al tráfico de un negocio de datos que se comercializan por completo, porque, a la final, los moradores digitales se cosifican y cuantifican como si de una especie de mercancía se tratara 9 .

En la banalidad que genera la promoción mercantilista de las redes sociales, y de acuerdo con Greenfield (2015), se desvela que la red causa conductas adictivas al punto de que en ciertas zonas cerebrales se causan deterioros en los mismos lugares que se ven afectados en los adictos a las drogas, razón suficiente para encasillar al Adán digitalis como un paciente capitalista y "fármaco-ponográfico" (Preciado, 2008, 2010). En igual sentido, entre los efectos, se destaca una importante reducción de materia blanca en regiones donde se controlan las emociones, capacidad de concentración y, en general, los efectos patológicos del IFS que fueron descritos con antelación.

En el mismo sentido, estudios de la University of Bergen (2014) muestran que el internet y uso de los dispositivos fármaco-pornográficos pueden incidir en la activación de zonas cerebrales relacionadas con comportamientos compulsivos. Ello podría explicar la razón por la cual, por ejemplo, el uso, muchas veces inconsciente, del smartphone lleva a

\footnotetext{
${ }^{9}$ Acxiom es una empresa de datos, comercia con información personal (datos) de aproximadamente trescientos millones de ciudadanos estadounidenses, llegando a saber más de cada uno que incluso el propio FBI. Además, los segmenta por categorías, ofertándolos en un catálogo, y aquellos que carecen de valor, en comparación con otros, los segmentan en la categoría waste (basura). Por su parte, los shooting star están conformados por pobladores en un rango de edad de 36 a 45 años, sin hijos, eventualmente casados y con una frecuencia deportiva de importancia, incluso, con gustos televisivos muy definidos.
}

Via Inveniendi Et Iudicandi

e-ISSN: 1909-0528 | DOI: https://doi.org/10.15332/19090528

Vol. 16 N.0 2 | julio-diciembre del 2021 
mantener conexiones permanentes con las redes sociales, ofreciendo, sin mayor esfuerzo, una especie de "satisfacción" inmediata que implica a nivel cerebral la restructuración de conexiones neuronales que buscan o aspiran a satisfacer el deseo hedonista de sentir placer; condición que dichos patrones comportamentales han aparecido en pacientes adictos a la cocaína ${ }^{10}$ : "cerca del 70 \% de los usuarios de Facebook visita el sitio a diario y cuando deja de hacerlo siente ansiedad” (p. 132).

Dicho contexto causa en el Adán digitalis otras emociones como la ya mencionada patología narcisista, pero, además, la ira y depresión están a la orden del día, ya que, por ejemplo, en redes sociales el autocontrol ${ }^{11} \mathrm{se}$ ve reducido y se cae, de manera sencilla, en un campo de desinhibición (Suler, 1996).

Fruto del contexto tratado, el filósofo coreano Han (2013) defiende el tránsito de la "sociedad disciplinaria de la prohibición" de Foucault (2002) a una nueva sociedad del rendimiento, que se caracteriza por técnicas novedosas de administración del tiempo y que dinamizan la atención desde el desarrollo de un Adán digitalis a quien le es sencillo acudir al multitasking.

De acuerdo con Han (2014b), la sociedad que se nos presenta en el día de hoy se identifica con el verbo modal positivo poder y su plural afirmativo y colectivo, yes, we can: "la sociedad disciplinaria todavía la rige el no y su

\footnotetext{
${ }^{10}$ Los estudios revelan que en quienes son adictos a Facebook aumenta la actividad cerebral en dos regiones específicas: la amígdala y el estriado. Estas regiones son a la vez capaz de contribuir a comportamientos impulsivos. El estudio descubrió que la principal diferencia entre un "adicto" y un "adicto a las drogas" es que el adicto a Facebook todavía conserva una actividad adecuada en la corteza prefrontal del cerebro, mientras que un drogadicto no lo hace (University of Bergen, 2014).

11 De manera específica, en Twitter, al menos el $46 \%$ de usuarios han admitido "trinar" con la finalidad de ventilar su ira o descontento con alguna situación. Esta conducta se conoce como mobbing.
} 
negatividad genera locos y criminales. Contrario sensu, en la sociedad de rendimiento colectivo, se producen depresivos y fracasados" (p. 89).

El Adán digitalis se concibe como un habitante puramente operacional (opera smartphones, display y medios tecnológicos), que permiten, de alguna manera, unirle a otros sin limitantes geográfico-temporales. Hoy en día existen los nómadas digitales, quienes, en su tránsito virtual constante y dinámico reducen sus problemas existenciales a una dicotomía básica: like /dislike.

Entonces, en el paraíso tecnológico, Adán está imbuido en un enjambre digital formado por individuos que no desarrollan unidad alguna dentro de una colectividad, caracterizados por la volatilidad en sus opiniones y comportamientos.

De este modo, los aparatos digitales traen una nueva coacción, una nueva esclavitud que, por demás, es de alguna manera voluntaria (autómata). Hay una explotación del ser humano de una forma mucho más eficiente en comparación a suplicios físicos y mentales, y, ahora, cualquier momento, espacio u oportunidad se presenta como propicia para buscar "estar conectados". De todas formas, es importante reconocer que "la comunicación digital hace que se erosione fuertemente la comunidad, el nosotros, destruye el espacio público y agudiza el aislamiento del hombre" (Han, 2014b).

Queda en evidencia que, así como la tecnología y su desarrollo en redes, se han convertido en la matriz poderosa de la que se desprende un sinfín de información que constantemente es supervisada y al mismo tiempo es mecanismo de control por la información suministrada de los usuarios en la red, esta herramienta tiene el poder de trasmitir conocimiento o ser utilizado como elemento estratégico que influye en el comportamiento de los sujetos pertenecientes a la sociedad.

Via Inveniendi Et Iudicandi

e-ISSN: 1909-0528 | DOI: https://doi.org/10.15332/19090528

Vol. 16 N.0 2 | julio-diciembre del 2021 
Es una realidad que en la red y en los muros de los lamentos digitales, las personas tienden a centrar sus conversaciones e interacciones, aproximadamente un $80 \%$, en sí mismos, generando conductas egocéntricas que propician, en todo caso, como se ha visto, sensaciones y emociones placenteras (so pena de caer en depresión). Dichos estímulos, como se ha expuesto, activan zonas cerebro-neuronales relacionadas con la adicción, los orgasmos, incluso, el mismo sentimiento de amor que se profesa hacia otra persona; $y$, en esta medida, las investigaciones que sustentan las afirmaciones lanzadas dejan en evidencia los focos de recompensa que recibe el cerebro cuando el Adán digitalis tiene protagonismo (Greenfield, 2015).

Parece entonces que las redes sociales y la tecnología hacen sentir al Adán digitalis libre, independiente y capaz de manifestarse sobre cualquier asunto sin caer o sentirse limitado por tabúes. Es una persona capaz de promover la revolución social más grande de la historia y, sin embargo, sucede todo lo contrario, ya que lo controlan, conocen lo que hace, sus gustos, preferencias, estado civil, lugares frecuentados, en suma, en virtud de la vigilancia tecnológica, todo se sabe (Assange, 2012).

Así, la dinámica que deja todo al descubierto lleva a un escenario panóptico, frente al cual, lo primero que conviene decir, tiene que "ver" con la pulsión de acumular y acumular información (Mendoza Perdomo et ál., 2016). Dichos comportamientos "fármaco-ponográficos" (Preciado, 2008) son característicos del capitalismo actual, ya que los dispositivos de vigilancia operan de forma pornográfica en una penetración hipervisual, la cual también había tratado Wajcman (2011).

En este orden de ideas, es viable concluir que la cara que se expone y solicita la atención a través de las redes sociales no es de ningún 
semblante, causando falta de identidad entre los moradores digitales (Non scoprire se libertá t’è cara ché il volto mio è carcere d'amore) ${ }^{12}$.

\section{Conclusiones}

Llegado este momento, es claro que las celdas, las estructuras físicas de vigilancia y represión han desaparecido y, en su lugar, los ideales de transparencia se han materializado. El tiempo y el espacio son meras quimeras que no limitan o cercenan el comportamiento de los moradores digitales y todo lo que les lleva a una actuación virtual les ubica en un campo en el que todo existe por siempre y vitalicio; nada corre el riesgo de envejecer, nada fenece, como bien lo expondría Saramago en Las intermitencias de la muerte.

Entonces, el ciberespacio es el hogar de lo digital y es allí donde las miradas se centran para determinar lo que en apariencia debe importar (y lo que no). La transparencia ha superado con creces los muros del panóptico benthamiano y se ha posado en cuerpos y mentes que actúan de manera autómata, aparentemente libre y, bajo la línea ilusoria del hedonismo.

De esta manera, en el panóptico digital, la diferencia entre vigilante y vigilado desaparece y el Adán digitalis disfruta de su desnudez en el paraíso electrónico, sin ser consciente de su mercantilización y reducción al nivel de ser, apenas, un dato más en el vertiginoso mundo de la red. Existe una vigilancia absoluta en la sociedad del hiperconsumo con fines de marketing, hasta el punto en el que los servidores conocen todas las preferencias y aficiones mejor que el Adán digitalis. La publicidad no es presentada de hecho en forma de anuncios, sino que se presenta como una

\footnotetext{
12 "No descubras si tienes en alta estima la libertad, pues mi rostro es la cárcel del amor" (Da Vinci, citado en Bredekamp, 2013).
}

Via Inveniendi Et Iudicandi 
especie de sugerencia magnífica que facilita la circulación del Homo digitalis por la selva de productos.

Es posible hablar hoy de una sociedad que se mueve en una época de las masas, tal y como lo entendió Le Bon (1995). En este sentido, el Homo digitalis se ubica en un panorama crítico en el que el pensamiento y conducta humanas (Arendt, 2009) se encuentran en vía de transformación, evidenciando una anarquía libertaria que centra su atención en las más superfluas banalidades que se promocionan a través del enjambre digital, afectando incluso la psiquis y con ella la estabilidad neuronal y emocional.

Dichos fenómenos de transición crítica llevan a tener como responsable a la misma revolución digital que está a la orden del día, aglutinando masas de Homo digitalis autómatas y aislados (hikikomoris). El Adán digitalis vive actualmente el dinámico desarrollo de las herramientas tecnológicas, las mismas que se diseñan y al mismo tiempo influyen en los usuarios de estas, respondiendo a un modelo global de comportamiento en la cual los hechos y las relaciones sociales mantienen una estrecha interrelación en la medida en que la velocidad con la que son comunicados los acerca o aleja cada vez más, generando con ello una cultura de dominación influida por la intercomunicación inmediata entre hikikomoris.

Actualmente, el Adán digitalis ya no es un mero receptor y consumidor pasivo de información, sino que, es un emisor y productor activo. Ya no basta consumir informaciones (de manera pasiva), sino que se quiere producir y comunicar activamente.

Esta función dual incrementa enormemente la cantidad de información. El medio digital no solo ofrece ventanas para la visión pasiva, sino también puertas a través de las cuales se lleva fuera las informaciones producidas por cada uno, cayendo en lo que se ha desvelado como una actual sociedad

Via Inveniendi Et Iudicandi

e-ISSN: 1909-0528 | DOI: https://doi.org/10.15332/19090528

Vol. 16 N.0 2 | julio-diciembre del 2021 
pornográfica y voyerista al renunciar a su esfera más íntima, resultado alarmante del estado de inconsciencia que modula la toma de decisiones y el mismo comportamiento.

Con lo mencionado resulta evidente que un verdadero proceso éticoeducativo no se encuentra ajeno a este proceso tecnológico-social-cultural. No puede cerrarse la posibilidad de que una herramienta como internet no se incorpore en la vida educativa bajo parámetros de responsabilidad, siendo digno de cuidado la incorporación de políticas educativas que incluyan estos cambios tecnológicos. En síntesis, es posible hablar de la existencia de un psicopoder que resulta muy eficiente al vigilar, controlar y mover a los hombres desde dentro, apoderandose de la conducta individual y social, toda vez que se engancha en el inconsciente. En este panorama, la sociedad de la vigilancia digital tiene acceso al inconsciente colectivo, ya que el comportamiento social (en masa) desarrolla rasgos igualitarios.

\section{Referencias}

Alonso, L. E. (2002). In Memoriam (1930-2002): Entre la bourdieumanía y la reconstrucción de la sociología europea. Revista Española de Investigaciones Sociológicas, 97, 9-28.

Arendt, H. (2002). Tiempos presentes. Gedisa.

Arendt, H. (2009). La condición humana. Paidós.

Assange, J. (2012). Freedom and the future of internet. BooksMobile.

Assange, J. (2014). Cuando Google encontró a Wikileaks. Clave Intellectual.

Barba, A. y Montes J. (2007). La ceremonia del porno. Anagrama.

Bauman, Z. y Lyons D. (2013). Vigilancia líquida. Paidós.

Baker, S. (2009). Numerati, Lo saben todo de ti. Seix Barral.

Via Inveniendi Et Iudicandi

e-ISSN: 1909-0528 | DOI: https://doi.org/10.15332/19090528

Vol. 16 N. 02 | julio-diciembre del 2021 
Bobbio, N. (1989). Estado, gobierno y Sociedad. Estado, gobierno y sociedad. Hacia una teoría de la política. Fondo de Cultura Económica.

Bentham, J. (2011). Panóptico. Círculo de Bellas Artes.

Bourdieu, P. (2000). Dominación masculina. Anagrama.

Bourdieu, P. (2011). Las estrategias la reproducción social. Siglo XXI.

Bukowski, Ch. (1978). La máquina de follar. Anagrama.

Da Vinci, L. (2013). Theorie des Bildakts. En Bredekamp. Rider University.

Foucault, M. (2002). Vigilar y castigar. Siglo XXI.

Galvis, L. y Pesca, D. A. (2020). Límites del tratamiento de los datos personales en el ámbito laboral frente al uso de las tecnologías de la información y comunicación en la era digital. Revista IUSTA, (52), 51-76.

https://doi.org/10.15332/25005286.5482

Greenfield, S. (2015). Your Brain on Social Media. Pentagrama.

Han, B. C. (2013). La sociedad de la transparencia. Herder.

Han, B. C. (2014a). En el enjambre. Herder.

Han, B. C. (2014b). Psicopolítica. Herder.

Harari, Y. N. (2014). De animales a dioses. Espa-Epulibre.

Huxley, A. (2013). Un mundo feliz. Cátedra.

Kafka, F. (1998). Cartas a Milena. Alianza.

Koerner, A. y Perafán Del Campo, E. A. (2020). Derecho social y tecnologías digitales. Via Inveniendi Et Iudicandi, 15(2), 249-276. https://doi.org/10.15332/625

Mendoza Perdomo, J. F., Amador Saavedra, J. A., Valencia Villamizar, D. y Carreño Dueñas, D. (2016). Derecho penal, vigilancia y control social. Ibáñez.

McLuhan, M. (1978). Wohin steuert die Welt? Massenmedien und Gesellschaftsstruktur. Verlag.

McLuhan, M. (1996). Comprender los medios de comunicación. Las extensiones del ser humano. Paidós.

Via Inveniendi Et Iudicandi

e-ISSN: 1909-0528 | DOI: https://doi.org/10.15332/19090528

Vol. 16 N.0 2 | julio-diciembre del 2021 
Moreno, C y Velásquez, A. (2015). Aspectos de la regulación TIC en Colombia. Revista IUSTA, (43). https://doi.org/10.15332/s1900-0448.2015.0043.01

Le Bon, G. (1995). Psicología de las masas. Morata.

Saramago, J. (2006). As Intermitencias Da Morte. Companhia das Letras.

Skype. (2013). Süddeutsche Zeitung Magazin. El Mundo.

Suler, J. (1996). The Psychology of Cyberspace. Rider University.

Parsons, T. (1952). The social system. Pentagrama.

Preciado, B. (2008). Testo Yonqui. Espasa.

Preciado, B. (2010). Pornotopía, arquitectura y sexualidad en "Playboy" durante la Guerra Fría. Anagrama.

University of Bergen. (Diciembre de 2014). Disabilty and Trauma. Psychological Reports.

Vitalis, A. M. (2015). De Orwell al cibercontrol. Gedisa.

Wajcman, G. (2011). El ojo absoluto. Manantial.

Via Inveniendi Et Iudicandi

e-ISSN: 1909-0528 | DOI: https://doi.org/10.15332/19090528

Vol. 16 N. 02 | julio-diciembre del 2021 